\title{
Carnets
}

Revue électronique d'études françaises de l'APEF

Deuxième série - 17 | 2019

Lîlle : prisme de la connaissance ou reconnaissance du monde

\section{Les possibilités d'une île : escale chez Marcu Biancarelli, Michel Houellebecq et Angelo Rinaldi}

\section{Ferdinand Laignier}

\section{(2) OpenEdition}

\section{Journals}

Édition électronique

URL : http://journals.openedition.org/carnets/10411

DOI : $10.4000 /$ carnets. 10411

ISSN : 1646-7698

Éditeur

APEF

Référence électronique

Ferdinand Laignier, «Les possibilités d'une île : escale chez Marcu Biancarelli, Michel Houellebecq et Angelo Rinaldi », Carnets [En ligne], Deuxième série - 17 | 2019, mis en ligne le 30 novembre 2019, consulté le 07 janvier 2020. URL : http://journals.openedition.org/carnets/10411 ; DOI : 10.4000/ carnets. 10411

Ce document a été généré automatiquement le 7 janvier 2020.

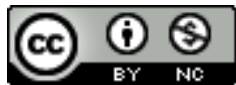

Carnets est mis à disposition selon les termes de la licence Creative Commons - Atribution - Pas d'utilisation commerciale 4.0 International. 


\title{
Les possibilités d'une île : escale chez Marcu Biancarelli, Michel Houellebecq et Angelo Rinaldi
}

\author{
Ferdinand Laignier
}

1 Bienheureuse, mortifère, isolée, déserte, paradisiaque, microcosme autonome et/ou utopique, fantasmatique, répulsive, les qualificatifs - et ceux-ci n'en sont qu'une petite partie - ne manquent pas pour désigner l'île, cet «espace à la fois ouvert sur l'infiniment grand, la mer, et refermé sur l'infini petit (...). Tiraill[é] entre le circonscrit et l'immense, (...) entre l'horizontal et le vertical» (Salini, 1999: 445). Fascinante et inspiratrice, l'insularité, véritable schème productif en littérature, se caractérise par le paradigme de l'identité topographique qui traduit le rapport entre l'espace et le réel. Pièce importante sur le damier du monde, l'île est un point d'ancrage et apparaît, dans un premier temps, dans l'œuvre de Marcu Biancarelli, de Michel Houellebecq et d'Angelo Rinaldi, comme un décor mobile, évolutif, mais surtout comme un espace actant $^{1}$, un miroir convexe des modèles sociaux, politiques, de la mémoire collective et une matrice d'émotions et de sensations. Réalité géographique, l'île n'en demeure pas moins investie par un imaginaire symbolique ancré dans les contextes socio-culturels, historiques et environnementaux qui font l'identité insulaire. Apostrophant tous les imaginaires du monde, l'île qui est à la fois l'ici et l'ailleurs et le lieu de rencontre entre l'imaginaire continental et la réalité insulaire, étonne, détonne et interroge le rapport de complémentarité entre perspectives géographique, humaine et littéraire. Si l'espace insulaire demeure le point constant de référence entre les trois œuvres, la représentation de l'île diffère. Le désir d'île biancarellien témoigne de l'enracinement culturel et affectif au lieu d'origine. Ce désir précède l'idée d'île symbolique et idéologique houellebecquienne, point de départ d'une représentation dystopique, construite sur une succession tragique d'événements impliquant le site insulaire dans son intégralité. Il devance également la négation rinaldienne de l'île, territoire replié sur ses bornes renvoyant au mythe de l'île étouffante et vénéneuse. Avec Murtoriu : Ballade des innocents, La Possibilité d'une île et La Maison des Atlantes, l'espace insulaire est mis en texte et le schème, en déployant "les arborescences de l'exil spatial et 
ontologique, du souvenir et de l'oubli, de la mémoire et de l'imagination, de la répétition et des rituels, des masques, des jeux et des dédoublements » (Isolery, 2014 : 205) de l'identité îlienne et son altérité non insulaire, structure la création artistique comme une nouvelle genèse. Le schème insulaire est alors une réponse de plus à l'interrogation de Jean-Marie Gustave Le Clézio: «L'insularité, au fond, qu'est-ce que c'est? C'est le lancinant désir de sortir de l'île à quoi tout horizon invite, de quelque côté qu'on se tourne. » (Le Clézio, 2008 : article en ligne) Si l'île conduit à réfléchir à la différenciation entre le dedans et le dehors, le schème, arche de Noé polychrome, polymorphe et polyvocale, pourfend sur une mer de certitudes, les notions de clôture et d'unicité pour s'aventurer dans les méandres de l'esprit et naviguer vers tous horizons dans l'aire/ère d'une île-monde.

\section{Pour une géographie littéraire...}

2 Au sein de Murtoriu: Ballade des innocents, La Possibilité d'une île et de La Maison des Atlantes, le logos et le topos n'ont de cesse de s'entrecroiser, de s'accorder pour exprimer à terme une géopoétique insulaire qui retranscrirait l'expérience de l'île par le biais de descriptions, de réflexions d'ordre géopolitique, de métaphores envahissant le texte. Ainsi la découverte, la redécouverte ou la mise en valeur de l'insularité répond à l'appel de cet espace, à « la spatialité que prend, revêt toute culture et, parallèlement, la façon, à un niveau individuel et symbolique, c'est-à-dire passée dans le langage, imaginée, la façon (...) dont l'homme exprime l'occupation de l'espace par son corps et les relations que le corps entretient avec l'espace, même à travers un texte littéraire " (Pageaux, 2000 : 132). À partir d'un espace référentiel - la Corse et Lanzarote - Marcu Biancarelli, Michel Houellebecq et Angelo Rinaldi souhaitent cartographier le réel, problématiser l'espace, et dépeindre une identité culturelle marquée par la relation au lieu insulaire sans s'interdire un imaginaire inauguré par l'introduction de nouvelles images pour créer un espace diégétique néanmoins crédible. Si ces écrivains ne peuvent être considérés comme des auteurs-géographes à part entière comme pouvait l'être Julien Gracq dont la production littéraire repose sur une fine description géographique, ils partagent la volonté de donner un sens au lieu, au rapport entre l'homme et la terre, à la représentation du paysage, et proposent une expérience du voyage, de l'altérité anthropologique en entraînant l'individu à «densifier sa sensation du monde au contact du lieu qu'il explore » (Roncato, 2008 : 79). Bertrand Westphal définit justement cette volonté de mettre en exergue le lieu habité et le rapport d'inclusion réciproque qui s'établit entre l'appropriation d'un pan du monde et la littérature :

N'est-il pas temps, en somme, de songer à articuler la littérature autour de ses relations à l'espace, de promouvoir une géocritique, poétique dont l'objet serait non pas l'examen des représentations de l'espace en littérature, mais plutôt celui des interactions entre espaces humains et littérature, et l'un des enjeux majeurs d'une contribution à la détermination ou à l'indétermination des identités culturelles? (Westphal, $2000: 17$ )

Le parcours des territoires romanesques biancarelliens, houellebecquiens et rinaldiens et la compréhension de la figure narrative que représente la logique de l'itinéraire géographique sont autant de manières significatives de reterritorialiser les personnages principaux que sont Marc-Antoine, Daniel1 et Tonio et dont le sentiment de présence au monde est plus que fragilisé. La représentation - ou la stylisation - des lieux par lesquels transitent les personnages témoigne d'un désir d'exprimer le réel en 
acceptant toutefois ses illusions et la réversibilité de ce dernier que suggère la littérature. L'image simonienne du gant retourné en est la meilleure illustration : «le monde à présent pour ainsi dire retourné à la façon d'un gant, d'un vêtement, révélé dans son envers ou plutôt perverti en ce sens que plus rien n'y avait la même signification, sinon de signification tout court " (Simon, 1981:426). L'identification d'un espace géographique dans sa globalité objective restituée dans un texte ne renvoie pas systématiquement à un espace matériellement existant d'autant plus que «la "réalité" n'appart[tient] définitivement à aucune perception particulière, qu'en ce sens elle est toujours plus loin»(Merleau-Ponty, 1964: 64). Par un arsenal de procédés détournés, d'allusions, d'impressions "invisibles", la littérature témoigne d'une "profondeur" et rend possible l'existence d'espaces vécus. L'espace littéraire ou espace "figuré", pour reprendre les propos de Roman Ingarden, se singularise de l'espace réel et de l'espace représenté par des manques, des omissions, des absences, des indéterminations : «Les espaces explicitement et réellement figurés sont (...) séparés comme par des lacunes (...) ; ils présentent pour ainsi dire des zones d'indétermination. Autant de situations qui sont tout à fait impossibles dans un espace réel. » (Ingarden, 1983 : 192) Hormis les fameux "trous noirs" dans l'espace... À la notion d'espace s'ajoute celle de paysage, définissable comme étendue terrestre délimitée par son horizon, qui tisse un rapport esthétique au territoire et qui devient ainsi objet où se fixe le regard qui lui donne naissance. Il reste également dépendant de sa représentation artistique. Comme l'affirmait Édouard Glissant : « le Paysage est en moi ce qui permet de m'égarer mais non pas de me perdre " (Glissant, 1981 : 261). Le paysage dans lequel l'individu projette une intention voire une intentionnalité est un carrefour entre l'intériorité de l'irreprésentable d'un soi qui se cherche pour se connecter au monde et l'extériorité de ce même monde qui attend d'être perçu. C'est le "processus" kantien même de formation du schème dans sa première phase. Cette rencontre soumise à l'autorité d'un regard imprégné de valeurs, de schémas, d'expériences vécues ou imaginées et de codes idéologiques interroge le paysage dans sa dimension phénoménologique où "[a]ucun objet n'existe en dehors du regard et des mots qui le constituent comme tel.» (Fougère, 2011: 183) Si l'espace se définit par une étendue, le paysage façonne un paradigme de la forme et, en étant tributaire de la dimension visuelle, fait appel à une large palette de schèmes émotionnels et esthétiques pour exprimer les interrelations qui se développent entre l'homme et son milieu. Les émotions naissent au croisement des paysages, des souvenirs, des expériences et des fantasmes. En poétisant le paysage, alors devenu source de projections, de sensations et d'émotions, en lui octroyant la possibilité d'être représenté sous forme d'images, ledit paysage, redevable d'une modalisation du regard et de sa dimension subjective en tant que perception personnelle et collective du lieu, implique l'observateur en provoquant son mécanisme de perception :

le paysage est l'affichage polysémique (conscient ou inconscient) dans un espace et dans un milieu donné, des projets, héritages, règles, bornes et dynamiques d'une société, qui permet le balisage et l'identification d'un territoire. Il exprime par là le fonctionnement d'un certain système socio-spatial; il est l'apparence construite d'une formation socio-spatiale donnée, à un moment donné, l'enregistreur des dynamiques et des dysfonctionnements présents et passés du complexe milieusociété. (...) De la sorte, il ne montre pas tout, il occulte des faits, voire déforme la réalité. Il n'est pas un simple miroir. (Morin, $2001: 325$ )

Murtoriu : Ballade des innocents et de La Maison des Atlantes offrent des paysages singularisant l'identité corse. C'est dans cet "écart" entre le regard subjectivant de 
Marcu Biancarelli et d'Angelo Rinaldi et le caractère d'une Corse dotée de qualités topographiques et environnementales que se forgent la narration d'une identité culturelle précise et la représentation d'une perception du monde permettant parallèlement une meilleure compréhension de l'espace dans lequel elle se forge. Partie de l'expression d'un sentiment intime du lieu corrélatif à la présence et au rôle actif du regard pour arriver à l'expression romanesque des manifestations, des spécificités, des symboles d'une culture insulaire, la Corse biancarellienne et rinaldienne, en déployant un imaginaire de la cohérence topographique, s'offre comme un vaste territoire à explorer poétiquement. La représentation d'un paysage, caractérisé comme la configuration d'un territoire, motive une interprétation créatrice et persuasive et lève des imaginaires de découverte, de singularité, de connaissance d'un lointain ou d'un proche. Ces imago mundi continueront de produire et de nourrir de nouveaux schèmes qui eux-mêmes généreront de nouveaux imaginaires essentiels à la représentation d'une insularité dans un rapport de contamination réciproque qui implique une réflexion sur les perceptions de l'espace. C'est exactement ce qu'Ernst Gombrich appelle le "schéma et sa correction", qui est au principe de toute l'évolution historique des arts, des sciences, etc. Ces

terrains de lecture : ce sont d'abord les œuvres mêmes où s'avance le lecteur, avec le sol de leurs mots, leurs reliefs de style, l'espace verbal, en somme, qu'elles ouvrent à la marche mentale - pensée, rêverie, désir. Mais d'autres ouvrages introduisent à une sorte de terrain aussi : ceux qui se fondent de manière plus forte, plus évidente, sur l'existence d'un rapport singulier avec ce que Merleau-Ponty nommait la chair du monde. (Richard, 1996 : 9)

Les interrogations qui entourent la notion de paysage trouvent une résonance dans la problématique qui résulte de la compréhension du visage. Si le jeu de l'homophonie poétique prête à sourire, le premier corollaire serait la perception visuelle consubstantielle à la reconnaissance des deux notions. Le paysage ne pourrait-il pas être considéré, perçu, révélé comme le visage d'un pays? La singularité du visage renvoie à la singularité du paysage et tous deux apparaissent dans une sensation d'immédiateté qui remonte en réalité depuis l'arrière-plan du schème et l'infinité de ses expériences et actualisations vécues. Sans doute est-ce pour cette double raison que « les visages ne sont pas simples, ils sont quasiment insondables, comme les paysages... et, si le visage était tout simplement le paysage d'une personne?» (Donadieu \& Périgord, $2005: 43)$ La rencontre du paysage mais aussi celle du visage est singulière et effective et invite l'altérité non pas à traverser mais à demeurer, à co-habiter. Contemplés, aperçus ou devinés, le paysage et le visage sont parcourus de mille et une nuances qui ouvrent les portes à l'imaginaire. David Le Breton rappelle que "[1]e corps est un filtre sémantique. Nos perceptions sensorielles, enchevêtrées à des significations, dessinent les limites fluctuantes de l'environnement où nous vivons. » (Le Breton, 2006: 19) Les coteaux en pente sont autant de rides qui se dessinent. Le visage et le paysage cachent autant qu'ils révèlent et l'on ne s'empare pas plus de l'un que de l'autre, tout juste leur reconnaît-on une mise en tension toujours génératrice d'une révélation (ontologique ?). Stéphane Mallarmé ne se contente pas d'apparier les sons mais cherche à fixer, dans le cadre ouvert par l'écriture, le schème de la beauté et de l'amour en transcendant l'humain de l'être (âme et/ou conscience de soi) jusqu'au seuil de l'entre (l'imaginaire et la seule réalité) : « Nous promenions notre visage / (Nous fûmes deux, je le maintiens) / Sur maints charmes de paysage, / o sœur, y comparant les tiens. » (Mallarmé, $1948: 58$ ) 


\section{La géologie insulaire ou les premiers pas du géographe}

6 Si Marcu Biancarelli et Angelo Rinaldi écrivent sur l'île depuis l'île et que Michel Houellebecq en propose une vision extérieure et continentale, leur complémentarité se lit au travers des éléments constitutifs de l'univers insulaire où la morphologie de l'espace insulaire s'exprime parallèlement à la morphologie du roman même. Murtoriu : Ballade des innocents et La Possibilité d'une île sont composés de "chapitres-îlots" au sein d'un ensemble plus vaste, le roman qui, comme l'île, est une image de totalité en réduction. Dans La Maison des Atlantes, les souvenirs de Tonio et leur enchaînement fractionné, tel un miroir brisé, sont autant d'îlots à la surface de la mémoire où le lecteur s'échoue et d'où il retrace le trauma originel du personnage rinaldien au reflet anamorphosé. La narration morcelée se fait donc territoire archipélique et le roman, tout comme l'île, rappelle les interrogations qui s'articulent autour des concepts de liaison/déliaison, de coupure/seuil, de claustration/liberté. Ce qui compose ou forme ne peut être que fragmentaire et la pensée de Pascal Quignard abonde dans ce sens : « tout à coup un fragment d'acier aimante mille fragments de tout ce qui nous entoure et qui est épars. (...) tout se polarise et fait récit. » (Quignard, 1990 : 63)

7 Le qualificatif de voyageur semblant exagéré pour caractériser Marc-Antoine, lui attribuer celui de promeneur n'est pas totalement déraisonnable : « À présent le terrain est plat. Le sentier se fait plus étroit, étouffé par des arbousiers et de gigantesques bruyères. Nous marchons silencieusement sans voir grand-chose tellement le maquis est dense.» (Biancarelli, 2012: 87) Marc-Antoine en arpentant "un sentier en forêt pendant une heure et demie pour rejoindre la rivière en contrebas, à un endroit où elle fait un coude, avec un imposant rocher dominant une large étendue d'eau » (ibid. : 86) se fait marcheur et invite implicitement le lecteur à la découverte de ce territoire rural, éloigné des axes de communication et préservé des défigurations urbaines. Le sentiment primitif, instinctif, du lien entre l'homme et le milieu détermine ainsi un espace associé aux racines, à l'autochtonie, aux valeurs d'authenticité et de convivialité. Sa temporalité distinctive est particulièrement régie par celle de la sensation allongée et alanguie : «Je ne dormais pas, je somnolais, les yeux fermés, mon esprit vagabondant parmi ces lieux majestueux. Je ressentais une paix extraordinaire, ouvrant les yeux de temps en temps pour me repaitre de sensations et d'images grandioses.» (ibid. : 94) C'est dans le cadre d'une déambulation, dans l'action de se mouvoir, que s'opère le rapport à ce lieu difficile d'accès, stimulateur des désirs partiels du rêveur et d'un imaginaire de la découverte, où il serait possible de vivre une utopie à travers le retour à la terre. Marc-Antoine marque le sol insulaire de sa présence physique et s'investit dans une dimension psychologique et intimement personnelle jouissant de profondes énergies cosmiques ou telluriques. La zone visitée, métaphoriquement assimilée au cœur de l'île, pourrait être considérée comme une île dans l'île où la dense végétation, agissant comme un rempart face à l'urbanisation dévorante, insularise ladite zone. Lieu quasiment virginal, celle-ci atteste toutefois d'une présence humaine et d'un passé plus ou moins ancestral :

Le sentier débute dans une forêt de pins puis continue en zone de moyenne montagne au milieu des chênes. Il semble parfois que nous nous trouvons sur une route ancienne, des murs de pierres soutiennent encore un chemin raviné. 
- Tu vois, on passait ici avec des chariots avant, explique Trajan, les Italiens venaient extraire le charbon. (ibid. : 86)

Marc-Antoine semble habité d'un désir du terrain qu'il se plait à arpenter sans retenue, lui qui est à la fois partie prenante et dépendante du lieu que l'on découvre à ses côtés et qui nous révèle à nous-même. Un désir déclenché par «ce mouvement pendulaire qui passe du "voir" au "donner à voir" " provenant "d'une géographie concrète patiemment investie et subie» (Bouvier, 1989 : 178). La vue du paysage est le moment d'une expérience émotionnelle forte où l'imprégnation de l'affectif dans le perceptif permet à Marc-Antoine de vivre une intense façon d'être au monde :

Nous avons grimpé le long des éboulis pour déboucher sur un plateau à moitié envahi par les poireaux sauvages et l'herbe de l'Ascension. Des abords du plateau la vue plongeait sur la plaine tout entière. Devenue presque blanche, la mer, à l'horizon, allait se confondre avec le ciel. D'ici on pouvait voir pénétrer dans les eaux ensoleillées les contours des côtes du pays, les Terres... De cette hauteur, nous avions l'impression de dominer en maîtres toute la zone méridienne, tout en étant au centre. (Biancarelli, $2012:$ 93)

9 L'espace, à première vue clos et limité, débouche sur une immensité qui place MarcAntoine en "maître du monde" éphémère et l'interroge sur la relation entre le stable et le mobile, la tension entre l'ici et l'ailleurs et la découverte d'un espace ouvert comme une potentielle réponse à sa quête de liberté mentale. Cette expérience "sublime" (ibid. : 92) trouve un écho dans l'analyse de François-George Maugarlone qui synthétise toute la pensée de Maurice Merleau-Ponty : «Je suis au monde donc le monde n'est pas moi.» (Maugarlone, 2007 : 33) Le fait d'appartenir au monde, le fait d'inclure toutes les possibilités d'un surplomb, donc de voir les choses d'en haut, clairement, distinctement, de pouvoir les catégoriser tend le spectateur vers l'être-au-monde. Or, paradoxalement, puisque Marc-Antoine appartient au monde, puisque le monde le comprend, le monde lui est étranger. Marc-Antoine n'est pas de nulle part, il est de quelque part d'où les choses lui apparaissent d'un certain point de vue. Le monde est cette profondeur inépuisable des "esquisses" pour reprendre le terme husserlien. MarcAntoine est/a donc un point de vue sur la chose mais tout le reste lui échappe. L'avoir et l'être se confondent dans l'expérience de dépossession du sublime. De sa position d'observateur, la contemplation panoramique d'une image dont il n'existe aucun autre exemplaire révèle de fait la complexité de la dynamique spatiale qui apparait en filigrane. Marc-Antoine, en harmonie, donne l'illusion d'être au centre de l'île qui est elle-même, symboliquement, au centre du monde. Un espace en mouvement extérieur à Marc-Antoine voit la mer ronger la terre liant ces deux espaces. La perception de l'espace ou l'expérience du terrain par un Marc-Antoine sensible au silence des lieux pourrait être définie par ce que Charles Avocat nomme l'acte de paysage :

[1]e paysage renvoie au sujet qui l'appréhende (...). L'analyse paysagère représente donc le point de rencontre entre deux réalités totalement différentes: d'un côté, une (ou plusieurs) image(s) sensorielle(s) correspondant à notre "vision" du monde, c'est-à-dire filtrées par notre imaginaire, notre psychologie, nos expériences antérieures, notre esthétique, de l'autre une réalité physique, objective, tridimensionnelle, dont nous recherchons la formulation mathématique et abstraite (...) ; entre les deux, c'est-à-dire entre la subjectivité totale et l'objectivité absolue, (...) un paysage vécu, perçu, observable par tout un chacun, à la fois réalité d'une image et image d'une réalité. (Avocat, $1984: 14$ )

L'escapade sauvage de Marc-Antoine joue un rôle essentiel dans la valorisation patrimoniale, écologique, du territoire insulaire corse et dans le sentiment que le tout s'offre à lui et réciproquement. Marcu Biancarelli accentue cette relation-sensation en 
proposant un paysage polysensoriel qui traduit une présence au monde en expérimentant le réel par le trajet sensible :

les cimes des arbres apparaissant en contrebas de la terrasse, la crête des montagnes semblant défier l'orgueil céleste, le vol d'une buse et le chant des grillons sortant de leur torpeur hivernale, ces éboulis qui nous faisaient un berceau pour nous protéger du monde, les murs en ruine d'un ancien enclos à chèvres lové dans un petit col, ces autres pierres levées appuyées sur un rocher pour soutenir un ancien linteau et cette longue muraille que nous avions longée pour atteindre la terrasse et qui semblait ceindre tout le promontoire, enfin ces blocs imposants, sur la plus haute pointe du monticule, ultimes héritiers d'un temps révolu, lorsqu'ils constituaient les fondations d'une tour bâtie pour les géants. (Biancarelli, 2012 : 94-5)

11 N'étant plus ici dans un espace-mouvement, Marc-Antoine fait état d'une anthropologie historique, d'une connaissance fine du territoire, d'un attachement aux lieux et aux éléments de la nature où le temps immémorial et le savoir populaire apposent leur marque dans l'espace :

- Non, mon ami, c'est un très vieux casteddu, une forteresse. D'une époque beaucoup plus ancienne. Où vivaient peut-être nos ancêtres, oui en un sens : nos ancêtres d'il y a des milliers d'années. Mansuetu, il y avait des gens ici, ils y vivaient, c'est la vérité. Qui sait ce que ces montagnes ont entendu de rumeurs, d'éclats de voix, et du bonheur de ces gens ou de leur désespoir. Ces murailles que tu vois, ce sont des fortifications, elles ont été bâties pour la défense. C'était peutêtre nos ancêtres, ou peut-être que nos propres ancêtres les ont attaqués et vaincus pour envahir leur territoire. Qu'est-ce qu'on peut en savoir? La mort est venue les prendre, tous, et il y a longtemps. Mais c'était un endroit de vie, où on trimait et où on s'agitait. Sûrement qu'ils plantaient des céréales dans le creux du vallon, qu'un point d'eau, aujourd'hui asséché, leur permettait d'étancher leur soif. (ibid. : 97)

Reconstituer une généalogie identitaire mythique,

partir, emboîter le pas des bergers, c'est expérimenter un genre de panthéisme extrêmement païen et retrouver la trace des dieux anciens - dieux des carrefours et de la chance, de la fortune et de l'ivresse, de la fécondité et de la joie, dieux des routes et de la communication, de la nature et de la fatalité - et rompre les amarres avec les entraves et les servitudes du monde moderne. (Onfray, $2007: 15$ )

Contrairement au protagoniste biancarellien qui arpente les lieux sauvages et qui a un contact direct avec le terrain, mesurant par ses périples le monde dont il annonce le déclin, la découverte du territoire insulaire par Daniel1 implique une saisie globale de l'espace. Il se traduit diversement selon les échelles d'observation où le regard dénote aussi un mouvement de distanciation qui autorise le lieu à naître car la singulière beauté insulaire est toujours variable selon l'angle de vue : «Peu avant l'atterrissage nous survolâmes une zone volcanique aux roches torturées, d'un rouge sombre.» (Houellebecq, 2005: 224) La distance spatiale se fait ici le vecteur d'une présence extérieure à l'île dont l'apparence inquiétante crée une nébuleuse étrange et laisse libre cours à l'imagination :

Le paysage n'est pas un cercle fermé, mais un déploiement. Il n'est vraiment géographique que par ses prolongements, que par l'arrière-plan réel ou imaginaire que l'espace ouvre au-delà du regard. (...) Le paysage est une échappée vers toute la Terre, une fenêtre sur des possibilités illimitées : un horizon. Non une ligne fixe, mais un mouvement, un élan. (Dardel, 1952 : 42)

14 L'éloignement physique et/ou culturel de Daniel1 marque la discontinuité de l'espace insulaire qu'il est en passe d'apprivoiser, sinon une cartographie mentale qui passe par 
cette suspension temporelle qu'impose l'avion, étape entre le continent et l'île, survolant la surface du territoire :

Pour imaginer, il faut de la distance entre soi et le monde, il faut du vide entre les choses, du néant à l'intérieur de l'être. (...) La carte a quelque chose à voir avec ce vide, ce qu'ont bien su repérer et traduire certains artistes en tentant de cartographier cet "entre", ou ce blanc, cet interstice. (...) Cet entre-deux (...) est une sorte de suspension, comme une parenthèse dans une phrase qui n'est pas la fin de la pensée, mais la condition au contraire de son exercice. (Tiberghien, 2007 : 132-4)

Michel Houellebecq s'est intéressé à la géologie de Lanzarote pour retranscrire une certaine atmosphère et n'a pas hésité, dans un souci de retranscription d'une ambiance climatique aride, à piocher dans la culture géomorphologique pour alimenter son œuvre : «Nous roulions dans une plaine d'un noir intense, presque bleuté, formée de rocs anguleux, grossiers, à peine modelés par l'érosion » (Houellebecq, $2005: 224)$. Ici, le territoire insulaire est un espace nu, rocheux qui renvoie une image de dépouillement. En ayant recours à une forme de "topo-démiurgie" Michel Houellebecq charge d'émotions la plage de Lanzarote. Daniel1, en solitaire mélancolique, ressent la pesée du milieu sur l'âme :

Peu avant d'arriver nous longeâmes une plage de sable noir, parsemée de petits cailloux blancs; je dois reconnaître que c'était étrange, et même perturbant. Je regardai d'abord avec attention, puis détournai la tête ; je me sentais un peu choqué par cette brutale inversion des valeurs. Si la mer avait été rouge, j'aurais sans doute pu l'admettre ; mais elle était toujours aussi bleue, désespérément. (ibid. : 225)

Le cadre étant posé, la plage qui, d'ordinaire, véhicule l'image d'un paradis estival, est énigmatique. La particularité géographique bouleverse l'habitude et Daniel1, vraisemblablement déstabilisé par ce "non-sens", projette ses craintes personnelles dans cet environnement insulaire proche d'un monde surréel. Lorsque Daniel1 décrit une autre plage avec une terminologie propre au discours géographique, il n'omet pas d'offrir son ressenti sur ce que le paysage lui inspire afin d'en préciser la physionomie et la structure. L'espace sollicite la spécificité de la vision houellebecquienne et de l'expérience du monde au travers du regard de Daniel1 qui tente de l'interpréter :

C'était une plage splendide, presque toujours déserte, d'une platitude géométrique, au sable immaculé, environnée de falaises aux parois verticales d'un noir éclatant; un homme doté d'un réel tempérament artistique aurait sans doute pu mettre à profit cette solitude, cette beauté. Pour ma part, je me sentais face à l'infini comme une puce sur une toile cirée. Toute cette beauté, ce sublime géologique, je n'en avais en fin de compte rien à foutre, je les trouvais même vaguement menaçants. "Le monde n'est pas un panorama ", note sèchement Schopenhauer. (ibid. : 106-7)

Daniel1 semble dans un rapport passif à l'espace, pire, il n'éprouve que de l'indifférence face à lui et n'a aucune réaction affective, se rangeant du côté de la sentence schopenhauerienne (Daniel1 ne semble pas jouir de la beauté du spectacle du panorama à la fois comme représentation et volonté). Le panorama, paysage élargi par définition, bout-du-monde par essence, s'offre à l'observateur tel un "être au lointain qui le rejoint là où il apparaît » (Merleau-Ponty, 1993 : 307). Mais Daniel1, comme en amitié, ratera cette rencontre...

18 Daniel25 fait état d'un monde déchiqueté dont la continuité territoriale a volé en éclats et c'est sa volonté de retourner au monde, délaissant l'espace confiné des néo-humains, cet appel du dehors qui fonde la reconnaissance du géographique insulaire :

Aujourd'hui le paysage d'immeubles calcinés, éventrés, les tas de gravats et de poussière produisaient une impression apaisante, invitaient à un détachement 
triste, dans leur dégradé de gris sombre. La vue qui s'offrait à moi était à peu près la même dans toutes les directions ; mais je savais qu'en direction du Sud-Ouest, une fois la faille franchie, à la hauteur de Leganes ou peut-être de Fuenlabrada, j'allais devoir aborder la traversée du Grand Espace Gris. L'Estrémadure, le Portugal avaient disparu en tant que régions différenciées. La succession d'explosions nucléaires, de raz de marée, de cyclones qui avaient déferlé sur cette zone géographique pendant plusieurs siècles avaient fini par araser complètement sa surface et par la transformer en un vaste plan incliné, de déclivité faible, qui apparaissait sur les photos satellite comme uniformément composé de cendres pulvérulentes d'un gris très clair. Ce plan incliné continuait sur environ deux mille cinq cents kilomètres avant de déboucher sur une région du monde mal connue, au ciel presque continuellement saturé de nébulosités et de vapeurs, située à l'emplacement des anciennes îles Canaries. Gênées par la couche nuageuse, les rares observations satellite disponibles étaient peu fiables. Lanzarote pouvait être demeurée une presqu'île, être devenue une île, ou avoir complètement disparu; telles étaient, sur le plan géographique, les données de mon voyage. (Houellebecq, 2005 : 462)

19 L'œuvre houellebecquienne met également en scène un archétype géographique qui n'est autre que l'île volcanique. Le volcan, force géologique fascinante, condensant les forces cosmiques, demeure le socle d'élaborations mythiques et poétiques et projette un imaginaire qui invite à la rêverie mêlant à la fois l'histoire de l'humanité et l'histoire de la Terre racontées par Daniel25 :

Lanzarote ; cette zone de l'Atlantique Nord, m'apprit-elle, avait connu un destin géologique tourmenté : après avoir été entièrement engloutie au moment de la Première Diminution, l'île avait ressurgi sous l'effet de nouvelles éruptions volcaniques; elle était devenue une presqu'île au moment du Grand Assèchement, et une étroite bande de terre la reliait toujours, selon les derniers relevés, à la côte africaine. (ibid. : 395)

Les métaphores du volcan, nombreuses, ambivalentes, laissent affleurer les forces telluriques, symbolisent la passion, participent à la création de réminiscences infernales, s'immiscent dans la vision mythique des origines et représentent le dynamisme de l'imagination créatrice d'une expression fantasmatique :

Analyser les figurations du volcan comporte ainsi des enjeux anthropologiques majeurs, éclairant le travail de sémiotisation par lequel l'homme construit sa relation à l'espace géographique et naturel mais aussi conçoit sa relation au corps et au monde social, dans une intrication entre le réel et l'imaginaire. (Bertrand, $2005: 7)$

21 Le volcan houellebecquien habite l'île autant que ses protagonistes qui en foulent le sol. «Les premiers volcans, légèrement menaçants dans la lumière d'orage » (Houellebecq, 2005 : 272) sont les témoins d'un "devenir" géologique de l'île en apparaissant comme un conflit qui ouvre l'histoire de l'île et demeurent ainsi liés à la mémoire du lieu. D’ordinaire imprévisibles, brutaux, effrayants, symboliques, "[1]es volcans [qui] se découpaient dans le lointain, présences familières, presque rassurantes sous le ciel d'un bleu sombre » (ibid. : 301), se personnifient comme les gardiens vigilants de l'espace insulaire et jouent un rôle dans l'élaboration d'une matière-émotion ${ }^{2}$. Celle-ci n'est autre que le motif littéraire qui sert la narration houellebecquienne puisque la puissance terrifiante et sublime d'un volcan se prête facilement aux représentations du corps et touche à la sexualité. Le magma bouillonnant, au cœur du volcan, métaphoriquement comparable au désir ardent érotique, évoque la perspective élohimite d'un hédonisme radical entre les partenaires du jeu amoureux : 
« II y a des vibrations spéciales... C'est très spirituel, vraiment.» (...) dans les ouvrages New Age il est classiquement admis que les régions volcaniques sont parcourues de courants telluriques auxquels la plupart des mammifères - et en particulier les hommes - sont sensibles; ils sont censés, entre autres, inciter à la promiscuité sexuelle. "C'est cela, c'est cela... » fit Patrick, toujours avec extase, «nous sommes des fils du feu » (Houellebecq, 2005 : 224) éjaculatoire, extériorise la virilité du feu tout autant que celle de l'homme. La lave à l'image de la semence masculine est soumise à un mouvement ascensionnel qui projette en haut ce qui se trouve en bas. L'énergie éruptive à la puissance dynamique inonde le sol de Lanzarote, infiltre la surface interne de l'espace insulaire pour galvaniser l'homme alors en contact avec les énergies souterraines du lieu. Le réveil du potentiel endormi de l'individu alimente la finalité du projet élohimite qui exige l'épanouissement des personnes :

En termes simples, il incita les adeptes à prendre conscience de la terre sur laquelle s'appuyaient leurs corps, à imaginer l'énergie volcanique qui émanait de la terre, cette énergie incroyable, supérieure à celle des bombes atomiques les plus puissantes; à faire leur cette énergie, à l'incorporer à leurs corps, leurs corps destinés à l'immortalité. (Houellebecq, $2005: 243$ )

L'espace insulaire aride, devenu énergétiquement fertile, émotionnel, dégage une vitalité créatrice qui ne demande qu'à se libérer et le prophète appelle l'homme, au travers de cette aventure physique incandescente, à sortir de ses limites et à ressentir la terre dans sa naturalité géologique la plus élémentaire au détriment d'une société moderne, industrielle, mortuaire.

Le volcan n'est plus seulement un monstre cracheur de feu mais donne lieu à une "rhétorique du paysage". Il devient le point final d'un voyage initiatique qui a fait suite à de nombreuses pérégrinations intellectuelles, sentimentales, physiques dans cette région de déchirure géologique et permet par l'ignition de réaliser le fantasme d'autoengendrement. L'excuse présentée aux adeptes de l'élohimisme suite au meurtre de leur prophète, toutefois remplacé par son propre fils, renvoie irrémédiablement à l'un des dogmes fondamentaux de la théologie chrétienne qui a vu Jésus mort devenir le Christ ressuscité. Le prophète assassiné devient un phénix, oiseau fabuleux doué d'une longévité surnaturelle, car l'énergie destructrice du volcan est un fluide bénéfique au processus imaginaire d'immortalité : « Il s'était donc jeté dans un volcan, livrant au feu son corps physique vieillissant afin de renaître, au troisième jour, dans un corps rénové. » (ibid. : 286) Le volcan houellebecquien, véritable "bouche volcanique", figure un schème de l'avalement où le volcan, anthropophage, acteur d'une réalité naturelle extrême que l'homme ne peut dominer, purifie car le feu créateur et l'énergie brute donnent naissance. Un renouveau qui doit tendre à un autre mode d'être, de sentir, de penser et d'agir dans le monde.

Le territoire insulaire qu'investit Tonio, malgré une répulsion assumée, est à l'image de la narration de ses souvenirs mis en texte par Angelo Rinaldi, fragmenté. La discontinuité géographique insulaire se couple aux discontinuités narratives, sémantiques et syntaxiques. L'enchaînement des souvenirs ne suit pas une logique particulière mais n'entrave en rien l'avancée de la narration. Cette dernière circule alternant progression et régression dans une chronologie simple de l'évolution du protagoniste principal pour, à terme, en densifier le portrait. Si l'herméneutique se fait géologie, Tonio, le narrateur, endosse un rôle qui le situe entre le géographe et le 
spéléologue explorant l'espace insulaire par le biais d'une résurgence mémorielle. Le chemin parcouru, tant dans l'espace que dans le temps, initie deux mouvements sagittaux contraires dans lesquels Tonio trouve sa position : l'un vers l'avant et l'autre vers l'arrière. Au niveau temporel, cela se traduit par la remémoration d'une mémoire singulière et des souvenirs qui transportent le lecteur dans un jeu d'anticipationremémoration au sein des plis énigmatiques de l'existence du personnage rinaldien. Au niveau spatial, cela passe par l'envie irrépressible de quitter la Corse avant d'éprouver le désir du retour à la terre d'origine.

La succession des souvenirs, traces aussi fugaces que l'empreinte d'un pas dans le sable avant la vague, donne lieu à un véritable archipel typographique, l'espacement chronologique entre chaque souvenir, potentiellement assimilable à des îlots textuels constituant « le lieu par excellence de la dislocation temporelle, d'une ouverture fluide et dynamique du souvenir, grâce auxquelles on assiste à l'éclosion sur le vif de l'intimisme du sujet, aux éclats de révélation autoportraitique » (W.Francis, 2008 : 111), correspond métaphoriquement à la dispersion des îles à la surface de la mer. En souhaitant oublier son passé, Tonio voit la Corse engloutie mais avec la réminiscence, elle s'impose avec d'autant plus de force à l'esprit. Si la Corse rinaldienne implique la séquestration et la négativité, la narration, dernier souffle de vie d'un Tonio mourant, se veut énergique et génératrice d'un mouvement. Réfutant une linéarité spatiotemporelle en contant au fil de la mémoire son histoire passée, Angelo Rinaldi va, par la voix de Tonio, tisser un "text'île" dont le schème insulaire complexe se révèle maille par maille. La distinction et la hiérarchie entre narration et description s'évanouissent nouant de fait le paradigme descriptif à la géographie insulaire :

la ville s'étend en longueur, tout contre la montagne; dans sa plus grande profondeur, on n'a guère que quelques centaines de mètres à franchir pour se retrouver dans la campagne et, cependant, la proximité de celle-ci n'est indiquée dans l'air par aucun effluve, même quand le maquis a macéré sous le soleil pendant toute la journée et que, à la tombée du jour, la brise descend des hauteurs : l'été, elle n'imprègne l'atmosphère que d'un souffle de braise, lorsque les bergers allument ces incendies. (Rinaldi $1973: 217$ )

La Corse tant détestée devient une source d'inspiration, une matrice de l'écriture pour Angelo Rinaldi, non seulement parce que Tonio ne désire pas s'y enraciner mais aussi parce qu'elle bouleverse sa vision du monde. Cependant la résurgence du passé diffuse une nostalgie du rattachement au lieu d'appartenance :

On s'enfonce dans une végétation exceptionnellement haute, drue et que je qualifierai aussi de saine, à cause de son élan dans une nature où, généralement, elle ne fait que se tordre au ras du sol, repliée sur elle-même comme un malade. On se croirait transporté dans une terre plus paisible, au climat tempéré, où les choses viennent à maturité, s'accomplissent sans violence et, par endroits, soudain, tonne le ressac de la mer qui détruit l'illusion à coups de boutoir. Alors, les cannes, empennées comme des lances de parade, se courbent sous le vent aigrelet qui monte du rivage oublié et annonce l'aube au-dessus de l'archipel toscan, dont les plaques sombres sont les derniers bastions de la nuit. On frissonne mais c'est de plaisir. (ibid. : 161-2)

28 A l'opposé de l'île honnie, caricaturée, l'Italie, dans un premier temps, offre un spectacle de la terre qui ne laisse pas Tonio indifférent. Les paysages italiens qui défilent plongent le protagoniste rinaldien dans une ivresse grisante qui favorise la réaction affective du retour sur soi, du ressourcement, et qui lui confère une pleine sensation d'exister. L'horizontalité de la nature observée engendre la verticalité de 
l'esprit. La continuité du territoire italien représente une échappée belle qui l'oppose aux paradigmes de l'espace et du temps erratiques insulaires. L'immobilité qui sclérose la Corse rinaldienne contraste avec le mouvement et la diversité qu'offre l'espace italien: "Nous marchions beaucoup, nous avons vu en Italie presque autant de villes que d'hôtels, pendant un mois. N'importe quelle ville, pourvu que l'on change. Nous établissions à minuit (...) des projets d'excursions dont nous savions bien que notre fantaisie les bouleverserait au réveil » (ibid. : 61). Tonio livre un espace mental et poétique en constante évolution d'autant plus que «ce qu'on nomme paysage se développe autour d'un point, en ondes ou vagues successives, pour se concentrer à nouveau sur cet unique objet, reflet où viennent se prendre tout à la fois la lumière, l'odeur, ou la mélancolie » (Cauquelin, 2011 : 13-4). Pour autant l'Italie, fascinante, quasi hypnotique, dont l'identité repose sur des caractéristiques géographiques, est un espace dans lequel Tonio pourtant ne souhaite pas vivre, se perdre, se retrouver, se dissoudre. En définitive il ne peut faire de cet espace le territoire où le soi se révèlerait en un "chez soi" :

Venise, je te la décrirais moins bien que mon aïeule vocératrice qui n'était pas sortie de sa montagne. Monuments que je photographie sous tous les angles, églises que je visite le nez en l'air, pour noter le détail d'une fresque, beaux panoramas, ils ne me laisseront aucun souvenir durable, et je confondrais les villes entre elles si des visages ne m'aidaient pas à les distinguer - peu sensible comme je le suis à la beauté des pierres et des monuments. (Rinaldi, $1973: 67$ )

L'affect de Tonio pour l'espace italien, pourtant proche géographiquement et culturellement $\mathrm{du}$ territoire corse, est tout relatif et dénote, d'une part, une assimilation profonde des paysages qui offre des motivations aux comportements du protagoniste rinaldien qui y circule et montre, d'autre part, que "[l]e rapport de l'homme à l'espace est de deux natures, l'une en relation avec la grandeur et la tentative de la maîtriser, l'autre en relation avec la forme du lieu, et la possibilité de la maîtriser cognitivement. » (Arnt, 2001 : 57)

30 L'espace et particulièrement l'insularité dans Murtoriu: Ballade des innocents, La Possibilité d'une île, et La Maison des Atlantes devient le motif de la structure textuelle si propice à l'approche géopoétique en exploitant le rapport au monde, au lieu, à la nature tout autant sur le plan de la réalité que de sa représentation. La démarche géopoétique de Marcu Biancarelli, de Michel Houellebecq et d'Angelo Rinaldi vise l'intégration de l'homme dans son milieu en n'omettant pas de redonner la parole aux lieux qui sont toujours chargés de mémoire historique, culturelle et socio-politique. $\mathrm{Si}$ l'appréhension de l'espace varie selon les protagonistes à cause de leurs expériences vécues, ils envisagent tous le rapport à l'espace en termes de mouvements: MarcAntoine ne voit sa pensée se dévoiler qu'en cheminant, Daniel1 et Daniel25 réfléchissent à se reconstruire dans un univers dilaté fait de découvertes, de contraintes assumées, gardant à l'esprit la notion de mobilité et Tonio cherche à s'émanciper définitivement de la terre qui l'a vu naître. Ces conceptions territoriales diffèrent du chronotope de l'ailleurs-maintenant biancarellien et houellebecquien et du chronotope de l'ici-autrefois du roman rinaldien. Comme le note Olivier Lazzarotti : « La consubstantialité de l'espace habité et de l'habitant constitue (...) le socle théorique de cette pensée dans laquelle nous nous reconnaissons ; ne pas traiter du monde et, secondairement, des hommes mais traiter des hommes à travers les interprétations du monde. » (Lazzarotti, 2006 : 170) En somme, la littérature ne doit pas renier cette part 
de territorialité, de chôra ${ }^{3}$, afin de rendre compte, toujours dans un souci de reproduction du réel, de ce rapport dialectique et plus essentiel de l'homme au monde.

\section{BIBLIOGRAPHIE}

ARNT, Héris (2001). « Espaces littéraires, espaces vécus », Sociétés, n 74, Louvain, De Boeck Supérieur, pp. 53-60.

AVocat, Charles (1984). «Essai de mise au point d'une méthode d'étude des paysages », in Charles Avocat (dir.), Lire le paysage, lire les paysages, Saint-Étienne, CIEREC, pp. 11-36.

BERQUE, Augustin (2009). Écoumène. Introduction à l'étude des milieux humains. Paris : Belin, « Belin poche ».

BERTRAND, Dominique (2005). «Introduction : Le pouvoir des métaphores telluriques : une "bouche d'ombre" politique? », in Dominique Bertrand (dir.), Nature et politique : Logique des métaphores telluriques. Clermont-Ferrand : Presses universitaires Blaise Pascal, coll.

«Volcaniques », pp. 7-13.

BIANCARELlI, Marcu (2012). Murtoriu : Ballade des innocents. trad. J. Ferrari, M-O. Ferrari, J.-F. Rosecchi, Arles : Actes Sud Éditions.

BOUVIER, Nicolas (1989). « Routes et déroutes. Réflexions sur l'espace et l'écriture », Revue des sciences humaines. Écriture et voyage, $\mathrm{n}^{\circ}$ 214, Paris, Avril-Juin, pp. 177-187.

CAUQUELIN, Anne (2011). L'Invention du paysage. Paris : PUF, coll. «Quadrige ».

COLLOT, Michel (1997). La Matière-émotion. Paris : PUF, coll. « Écritures ».

DARDEL, Éric (1952). L'Homme et la terre. Paris : PUF.

DONADIEU, Pierre, PERIGORD, Michel (2005). Clés pour le paysage. Paris : Ophrys Éditions, coll.

« Géophrys ».

FOUGÈRE, Éric (2011). La Littérature au gré du monde. Espace et réalité de Cervantes à Camus. Paris :

Classiques Garnier, coll. « Géographie du Monde ».

GLISSANT, Édouard (1981). Le Discours antillais. Paris : Seuil.

HOUELlebeCQ, Michel (2005). La Possibilité d'une île. Paris : Fayard.

INGARDEN, Roman (1983). L'Euvre d'art littéraire. Lausanne : L'âge d'homme, coll. « Slavica ».

ISOLERY, Jacques (2014). « De l'impossibilité d'une île et de la possibilité du livre : approche du schème insulaire dans l'œuvre de Jean Lods ", in Maria de Jésus Cabral, Ana Clara Santos (dir.) Les Possibilités d'une île. Paris : Éditions Petra, pp. 205-227.

LAZZAROTTI, Olivier (2006). Habiter, la condition géographique. Paris : Belin, coll. « Mappemonde ». LE BRETON, David (2006). « La conjugaison des sens : essai », Anthropologie et sociétés, vol. 30, n 3, pp. 19-28. 
LE CLÉZIO, Jean-Marie Gustave (2008). « Les mille et une îles de Le Clézio » par Jean-Louis Ézine, article en ligne (URL : http://bibliobs.nouvelobs.com/romans/20081009.BIB2168/les-mille-etune-iles-de-le-clezio.html)

MALLARMÉ, Stéphane (1948). Dix Poèmes de Stéphane Mallarmé. Genève : Droz, coll. « Textes

littéraires français ».

MAUGARLONE, François-Georges (2007). Retour à Merleau-Ponty. Paris : Grasset.

MERLEAU-PONTY, Maurice (1964). Le Visible et l'invisible. Paris : Gallimard, coll. « Tel ».

MERLEAU-PONTY, Maurice (1993). Phénoménologie de la perception. Paris : Gallimard, coll. « Tel ».

MORIN, Serge (2001). Les Montagnes tropicales. Identités, mutations, développement. Bordeaux : Presses Universitaires de Bordeaux, coll. « Espaces tropicaux ».

ONFRAY, Michel (2007). Théorie du voyage. Poétique de la géographie. Paris : Le Livre de Poche, coll. «Biblio Essais ».

PAGEAUX, Daniel-Henri (2000). « De la géo-critique à la géosymbolique. Regards sur un champ interdisciplinaire : littérature générale et comparée et géographie. ", in Bertrand Westphal (dir.) La Géocritique : mode d'emploi. Limoges : Presses universitaires de Limoges, pp. 125-160.

QUIGNARD, Pascal (1990). Petits traités II. Paris : Gallimard, coll. « Folio ».

RICHARD, Jean-Pierre (1996). Terrains de lecture. Paris : Gallimard, coll. « Blanche ».

RINALDI, Angelo (1973). La Maison des Atlantes. Paris : Gallimard, coll. « Folio ».

RONCATO, Christophe (2008). «L'atopie ou le processus de désencombrement. Une lecture de The Blue Road de Kenneth White ", Études écossaises, vol. 11, pp. 79-90.

SALINI, Dominique (1999). «L'île entre laboratoire et lab-oratoire ou la complexité de la démesure ", in Anne Mestersheim (dir.), L'île laboratoire. Ajaccio : Alain Piazzola, pp. 443-449.

SIMON, Claude (1981). Les Géorgiques. Paris : Éditions de Minuit.

TIBERGHIEN, Gilles (2007). Finis Terrae. Imaginaires et imaginations cartographiques. Paris : Bayard, coll. «Le rayon des curiosités ».

W. FRANCIS, Cécilia (2008). « Souvenir et fragment : le discours autobiographique chez Gabrielle Roy ", in Carlo Lavoie (dir.) Lire du fragment: analyses et procédés littéraires. Québec : Éditions Nota Bene, coll. « Terre américaine », pp. 95-114.

WESTPHAL, Bertrand (2000). « Pour une approche géocritique des textes », in Bertrand Westphal (dir.), La Géocritique : mode d'emploi. Limoges : Presses universitaires de Limoges, pp. 9-41.

\section{NOTES}

1. Né du schéma actantiel, l'espace actant se perçoit comme un personnage défini par sa fonction et son champ d'action mais également par le caractère multi-relationnel qu'il entretient avec les autres. On note également une dramatisation de l'espace insulaire qui accentue l'importance du lieu au sein des trois romans.

2. Voir Collot (1997).

3. Je pense notamment à la définition d'Augustin Berque. La chôra, créatrice des représentations, est à la fois empreinte et matrice de l'existence des choses à savoir un « lieu dynamique, à partir 
de quoi il advient quelque chose de différent, non pas un lieu qui enferme la chose dans l'identité de son être » (Berque, 2009 : 29).

\title{
RÉSUMÉS
}

Placé sous le signe du paradoxe, l'espace insulaire, singulier, différent, à part, demeure un territoire qui suscite de nombreuses réactions et se présente comme l'épicentre de l'imaginaire. À chaque vision auctoriale se dessine une appréhension de l'île. La Corse de Murtoriu : Ballade des innocents devient un espace temporaire pour un protagoniste qui pense de plus en plus à un départ, et qui se lasse du caractère autocentré de ses contemporains. Avec La Possibilité d'une île, Michel Houellebecq souligne la dimension libératrice de l'île, semblable, dans un premier temps, à un paradis terrestre retrouvé où la contagion de l'immortalité n'est plus un mythe. Mais l'utopie du post-humain vire à la dystopie. Enfin, La Maison des Atlantes d'Angelo Rinaldi dépeint une Corse étouffante, toxique, statique, figurant une île carcérale et stérile. Ces trois récits ouvrent la voie d'un dialogue interculturel sur les enjeux géoculturels, géoenvironnementaux et géohistoriques de la société insulaire.

Placed under the sign of paradox, the island space, singular, different, apart, remains a territory that arouses many reactions and presents itself as the epicentre of the imaginary. At each auctorial vision there is an apprehension of the island. The Corsica of Murtoriu: Ballad of the Innocent becomes a temporary space for a protagonist who thinks more and more of a departure, and who gets tired of the self-centred character of his contemporaries. With La Possibilité d'une île, Michel Houellebecq emphasizes the liberating dimension of the island, similar, at first, to a regained Paradise on Earth where the contagion of immortality is no longer a myth. But posthuman utopia becomes dystopia. Finally, La Maison des Atlantes by Angelo Rinaldi depicts a suffocating, toxic, static Corsica, depicting a sterile prison island. These three stories pave the way for intercultural dialogue on the geocultural, geoenvironmental and geohistoric issues of island society.

\section{INDEX}

Keywords : insularity, geopoetics, writing, island representation, space

Mots-clés : insularité, géopoétique, écriture, représentation de l'île, espace

\author{
AUTEUR \\ FERDINAND LAIGNIER \\ Université de Corse, UMR CNRS 6240 LISA \\ ferdinandlcc[at]yahoo.fr
}

